\title{
Computational Topology in Text Mining
}

\author{
Hubert Wagner, Paweł Dłotko, and Marian Mrozek \\ Institute of Computer Science Jagiellonian University, \\ \{hubert.wagner, pawel.dlotko,marian.mrozek\}@ii.uj.edu.pl
}

\begin{abstract}
In this paper we present our ongoing research on applying computational topology to analysis of structure of similarities within a collection of text documents. Our work is on the fringe between text mining and computational topology, and we describe techniques from each of these disciplines. We transform text documents to the so-called vector space model, which is often used in text mining. This representation is suitable for topological computations. We compute homology, using discrete Morse theory, and persistent homology of the Flag complex built from the point-cloud representing the input data. Since the space is highdimensional, many difficulties appear. We describe how we tackle these problems and point out what challenges are still to be solved.
\end{abstract}

Keywords: Computational topology, Computational homology, Flag Complex, Discrete Morse theory, Text mining, Vector space model.

\section{Introduction and Existing Work}

With the growth of the Internet, efficient and accurate information-retrieval systems are of great importance. Modern search-engines are able to quickly query amounts of data counted in exabytes. Text mining aims at performing more indepth analysis, revealing some additional knowledge from the data.

Text mining methods often use graph-theoretical approaches [11. Analysing the connected components of the graph of similarities between pairs of documents is a simple example. From a topological perspective, such analysis operates on 1-dimensional complexes (only pairs of documents are considered) and gives 0-dimensional topological information.

In general, higher dimensional relationships, i.e. relationships between larger subsets of data, are sometimes used in data-mining. For example, the number of triangles (3-cliques) is an important descriptor of the connectivity of a social or collaborative network [6. Rather than finding just the number of such higherdimensional elements, we would like to compute their topological structure.

We believe that mining a higher dimensional topological structure within a set of text documents can give an important insight into the data. In general, the current state-of-the-art topological methods are incapable of handling large datasets in high dimensions, but efficient methods are being developed [15]. Still, we believe that experimenting with smaller, properly sampled data can give interesting insights. For example, [3] shows that data coming from natural images form a topological Klein bottle. 
In the ongoing research, done in cooperation with Google, we use the tools of computational topology to robustly analyse and compare text data. The goal is to find meaningful topological patterns. This information can help understand the global structure of the data. In a longer perspective, this knowledge can be used in conjunction with the standard methods, improving the quality of information-retrieval systems. This is a novel direction, as is the application of computational topology in higher dimensions. In this paper we show how we adapt existing topological methods and how we tackle computational difficulties, exploiting certain properties of the data. The main question we seek to answer is whether the current computational topology algorithms are capable of efficiently handling reasonable amounts of text data.

For an introduction to computational topology see [5]. A paper by Carlson [3] is an important work, which shows that analysis of higher-dimensional data can be meaningful. A number of papers dealing with lower-dimensional spaces exist, but these techniques are hard or impossible to generalize to higher dimensions [12. A recent paper by Zomorodian [15] deals with building Rips complexes of high dimensional data, which is also part of our computations. A PhD thesis of Lewiner 9 describes the usage of discrete Morse theory to compute homology groups.

\section{Background}

\subsection{Vector Space Model}

We start with describing a way to map textual data into a representation which allows us to use topological tools. Vector space model is a standard tool in information retrieval and data mining [13. A corpus, i.e. collection of text documents, is mapped into points (or vectors) in $\mathbb{R}^{n}$. These vectors are the so-called termvectors and each of them represents a single document, as described below. Each dimension in this space corresponds to a single word (or term).

With each document in a corpus, we associate a term-vector [13, containing words characteristic of this document. In practice from 10 to 50 words are extracted. While term-vectors do not fully describe the documents, they roughly encapsulate the topic. Each term $t$ contained in some document $d$ in corpus $D$ is weighted according to the standard $t f$-idf 13 technique: $w(d, t)=$ $t f(d, t) \cdot i d f(t)$, where $t f(d, t)$ is the number of occurrences of word $t$ in document $d$, and $i d f(t)=\log \frac{|D|}{|\{d: t \in d\}|}$. Thus, more frequent words in a document are weighted higher but this is offset by the global popularity of a given term. By $P$ we denote the array of term-vectors representing all the documents of the corpus $D$. Each term-vector is associated with a unique integer, which is the index of that term-vector in $P$.

In terms of implementation, each term in the corpus can also be assigned a unique number, which represents the term. This is more efficient than storing multiple copies of the string representations of the terms. Term-vector $d \in P$ is compactly stored as a sparse vector: we explicitly represent only the coordinates 


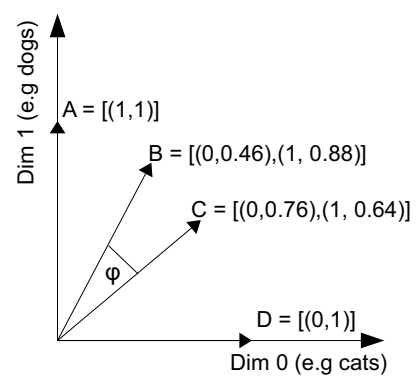

Fig. 1. Example of the vector space model. A two-dimensional space is shown, which means that only two different words are extracted from all documents. The similarity between vectors $B, C$ equals $\cos (\varphi)=0.46 \cdot 0.76+0.88 \cdot 0.64=0.91$.

with non-zero weights. The actual data-structure representing term-vector $d$ is simply an array of pairs (index of $t, w(d, t)$ ). See Figure 1 for a simple example. Note that, for brevity, we often identify a document with its term-vector.

Rather than using the Euclidean metric on this space, we use the so-called cosine similarity measure. This is a natural choice, as this measure is a standard text mining tool used to compare documents. The similarity between two documents (represented by term-vectors $a, b)$, is given by $\operatorname{sim}(a, b):=\cos (\angle(a, b))=$ $\frac{\langle a, b\rangle}{\|a\||| b \|}$. This formula requires computing square roots, which is costly. We will store normalized (according to Euclidean norm) term-vectors and equivalently compute similarity as:

$$
\operatorname{sim}(a, b)=\langle a, b\rangle
$$

Cosine similarity quantifies the closeness of topics of two documents [13. The values range from 0 (completely unrelated topics) to 1 (identical topic). Note that the constructed space (equipped with the cosine similarity measure) is not a metric space. Later we will use a weight function $d(a, b):=1-\operatorname{sim}(a, b)$, which is also not a metric, as the triangle inequality is not satisfied.

We have to distinguish between extrinsic (embedding) and intrinsic dimension of the space. In this case, the extrinsic dimension, $\mathrm{R}$, is large, equal to the number of unique words in the dataset, which can reach tens of thousands to several millions in practical applications. It is typically assumed that the intrinsic dimension is significantly lower, which prevents the curse of dimensionality from making the computations infeasible. Another important property of data coming from real-world text corpora is that the frequency of word occurrences follow the Zipf distribution 14. It assumes that the frequency of an $r$-th most common word is expressed as: $P(r)=\frac{R}{r \ln (c R)}$, where $c$ is some constant which depends on the corpus. This distribution is far from uniform - intuitively, the most common words apear much more often than the others.

Also, note that due to very high extrinsic dimensionality, the space is very sparse (empty) in practice. Another observation is that the number of keywords 
extracted from each document is relatively small. In practice, this number is chosen between 10-50. So, for each term-vector, the number of nonzero coordinates is small, compared to the number of zero coordinates. Therefore, the similarity between two randomly chosen term-vectors should be zero most of the time, since the support of these vectors is disjoint. These facts suggest that this space behaves differently than the Euclidean space, where the distance between any two points is finite (intuitively, zero similarity corresponds to infinite distance). In practice, this effect is offset by the Zipf distribution - more popular words increase the number of pairs of documents with nonzero similarity.

The described properties of the data are important, as they reduce the number of large cliques appearing during computations. This makes topological computations based on flag complexes, as described in the following section, more feasible.

\subsection{Computational Topology}

First, we would like to outline the computations we perform. We are interested in computing homology and persistent homology of the space describing similarities between the documents in a corpus. Representing the textual data in the vector space model yields a point-cloud, allowing us to use topological tools. Starting from the point-cloud we will construct a simplicial complex called a flag complex, which encodes higher dimensional topological information, and can be viewed as a higher-dimensional analog of a graph. Since the complex can be large, we simplify it, using discrete Morse theory. This step retains the topological information. Finally, we compute homology on the reduced complex.

A finite collection of sets, $S$, is an abstract simplicial complex if for every $t \in S$ and for every $s \subset t$ we have $s \in S$. Every element $t \in S$ is a simplex and its dimension is defined as $\operatorname{card}(t)-1$. By $S_{k}$ we denote the $k$-skeleton of complex $S$, i.e. all simplices in $S$ with dimension $\leq k$. If $p \subset q$ and $\operatorname{card}(q)-\operatorname{card}(p)=1$, we say that $p$ is a face of $q$ and $q$ is a co-face of $p$. (Co-)boundary is the set of all (co-)faces of a simplex. A simplex of dimension $0,1,2$ is respectively: a vertex, an edge and a triangle.

An $\epsilon$-graph imposed by the similarity measure sim on the collection of termvectors $P$ is defined as $G=(P, E)$, where $E=\{(a, b) \in P \times P \mid 1-\operatorname{sim}(a, b) \leq \epsilon\}$. In other words, edges connect pairs of documents with similarity above certain threshold. In general, for graph $G=(V, E)$, a subset $V^{\prime} \subset V$ is a clique if for every $v_{1}, v_{2} \in V^{\prime},\left(v_{1}, v_{2}\right) \in E$. Flag complex of graph $G$ is defined as: $S(G):=\left\{V^{\prime} \subset V \mid V^{\prime}\right.$ is a clique in $\left.G\right\}$. The flag complex of a graph $G$ is the maximal simplicial complex having $G$ as its 1-skeleton.

\section{Construction of Flag Complex}

The flag complex, as well as the so-called Vietoris-Rips complex (see [5]), is a standard tool used to perform topological data analysis [3. In this section we describe an efficient bottom-up technique to obtain flag complexes. The presented technique avoids the usage of associative data structures, which incur 
a significant performance penalty. We designed the code to use only vectors (dynamically growing arrays as in the $\mathrm{C}++$ Standard Library) which are fast due to good caching properties.

The complex building phase is similar to the construction of Vietoris-Rips complex presented in [15. Since in Section 4 we are focused on computing Morse complexes, we require fast access to the (co-)boundary of each simplex, which is not included in the cited paper. We use the name flag complex instead of Vietoris-Rips complex, as the latter assumes a metric function.

The input to the algorithms presented in this Section is the array $P$ together with the similarity function sim. Let us first describe the data structure we use to store simplices. Each simplex $s$ has a vector vertices storing the 0-dimensional simplices (which correspond to indices of term-vectors) that belong to $s$. Moreover, it has a vector boundary, containing the faces of $s$.

During the construction we also use vectors coboundary and neigh. Vector $s . n e i g h$ contains the vertices adjacent to all vertices in $s$, with the additional property that for each $i \in s$.neigh $i>\max \{s$.vertices $\}$. We assume the the entire simplex is created by its maximum vertex. Importantly, we exploit this property in the algorithms to ensure that each simplex is created only once (when the maximal vertex is processed). SimplicialComplex stores a vector of pointers to simplices separately for each dimension. Algorithm 1 shows how we build the 1-skeleton of the constructed flag complex.

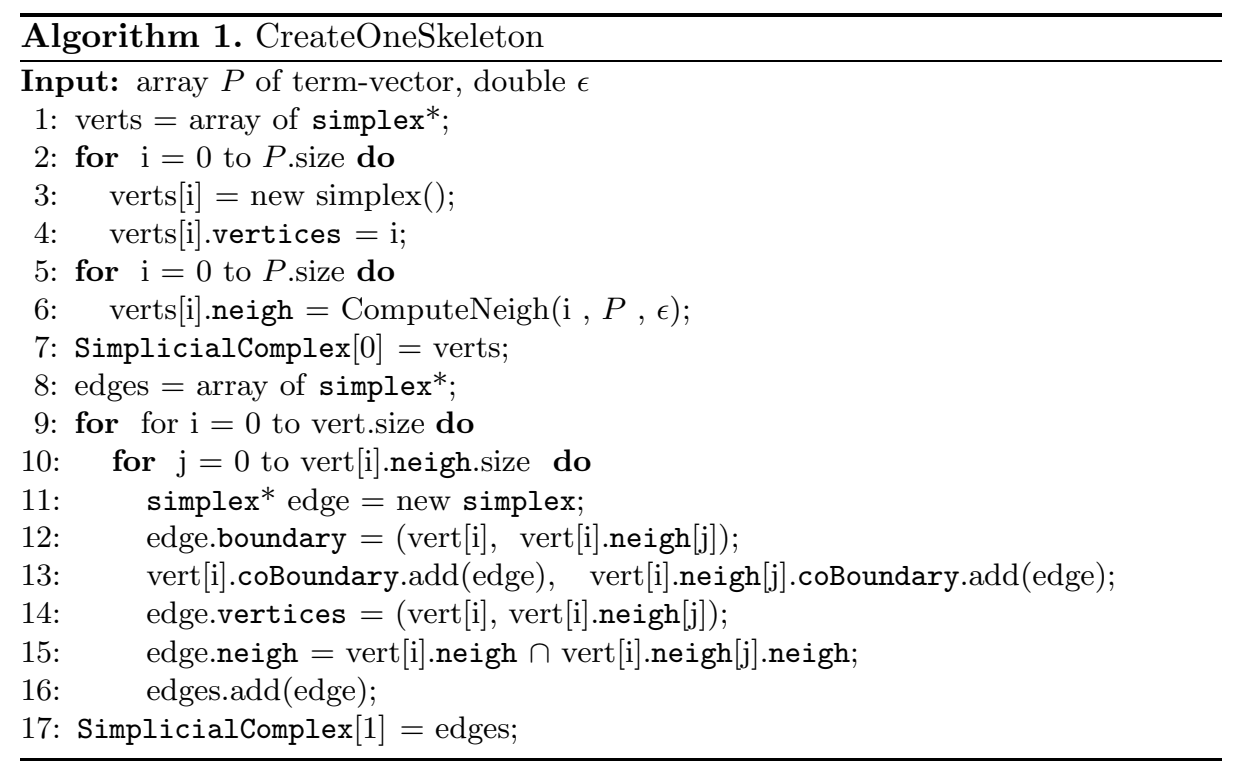

ComputeNeigh algorithm computes the $\epsilon$-neighborhood of a given vertex with a constraint that it returns only vertices with indices higher than the index of the considered vertex. For the time being, we assume that it just iterates through 
all the vertices, computes the similarity and rejects the vertices corresponding to documents with similarity below the threshold. This makes the complexity of the entire Algorithm 1 quadratic. Since, in practice, the output graph is sparse, the complexity can potentially be reduced. We are investigating methods of computing $\epsilon$-graphs, such as cover trees [2], which are more suitable for this type of data. Some efficient techniques for metric spaces are reviewed in [15].

Once the 1-skeleton of the complex is created, we proceed with the creation of higher dimensional simplexes, as described in Algorithm 2 In terms of computational complexity, the entire constructed flag complex can be exponential in the number of vertices of the input. This is related to the fact, that the total number of cliques is pessimistically exponential. In practice, we are interested in computing the complex only up to a certain, small dimension, which yields polynomial worst-case complexity. The actual performance is heavily dependent on the data.

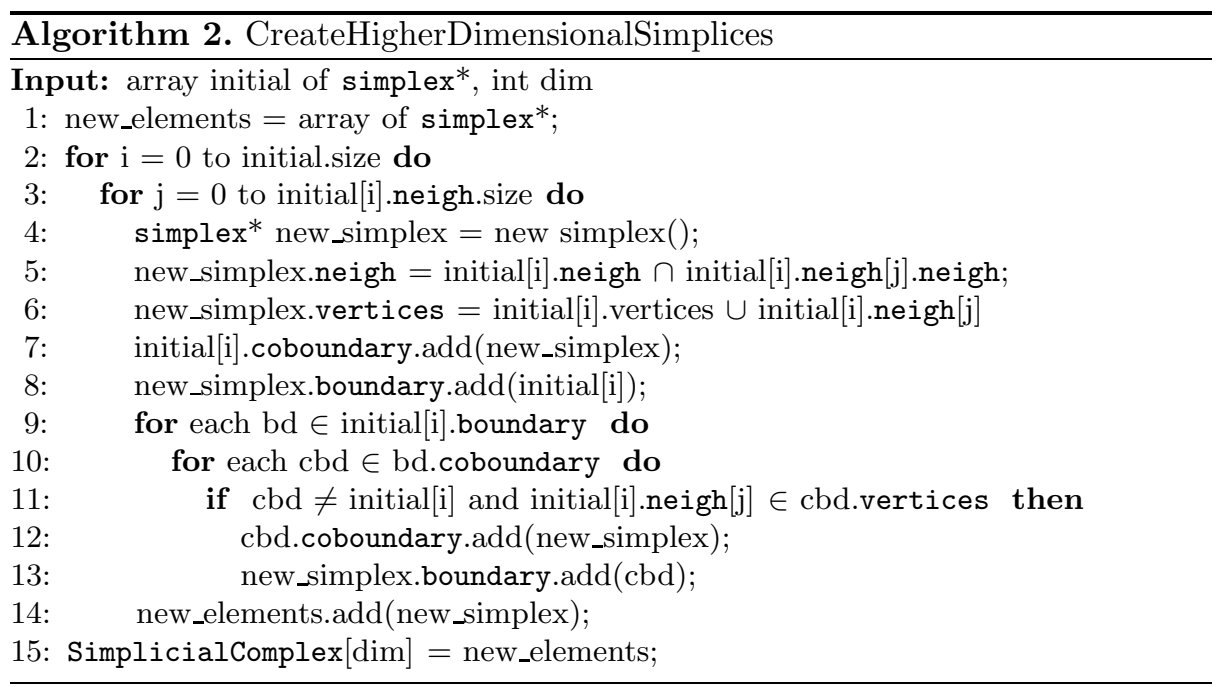

\section{Morse-Flag Complexes}

In this section we show how to use discrete Morse theory [7] to compress the Flag complex during its construction. In particular we introduce a novel algorithm which iterates Morse complex computations (see Algorithm 3), yielding the nonpersistent $\mathbb{Z}_{2}$ homology of the input complex (see Theorem 11). Exploiting this property, we do not have to generate boundary matrices required for algebraic computations, which tend to be costly in terms memory and time. To the best of our knowledge this is the first algorithm to compute homology in general dimension, which does not rely on matrix reduction. The obvious limitation is the fact that is requires field coefficients. 
To algorithm proceeds as follows: it computes a Morse matching, then computes the boundaries between the critical (unpaired) cells, which form a Morse complex. Now we repeat the process as long as there exist some nonzero boundaries, which allow to perform the Morse matching.

Note that using $\mathbb{Z}_{2}$, rather than $\mathbb{Z}$, coefficients simplifies the computations, but prevents us from capturing the so-called torsion (see [5]) in homology groups. In terms of implementation, during the construction in dimension $n$ we need to store only the $n-1$ and $n-2$ dimensional elements, which enables us to reduce the memory usage.

Theorem 1. Let $S$ be the input complex. We build a Morse complex of $S$ and iterate Morse construction, as long as some Morse pairings exist. Let $\left|S_{n}\right|$ denote the number of $n$-cells in the final Morse complex. Then $\operatorname{dim} H_{n}\left(S, \mathbb{Z}_{2}\right)=$ $\beta_{n}(S)=\left|S_{n}\right|$.

Proof. Since, in general, the Morse complex obtained in the described construction is not a simplicial complex, we use a more general algebraic Morse theory (see Section 11.3, 8]). Compared to the setting of Forman [7], the important difference is that during the matching between cells $a$ and $b$, where $a$ is a face of $b$, we want the incidence coefficient $(\kappa(a, b)$, see [8] ) to be invertible. However, in case of field coefficients, for $a$ being a face of $b$ we have $\kappa(a, b) \neq 0$, so it is invertible in the field.

We now show that iterative construction of Morse complexes terminates with complex whose all cells have empty boundaries (and consequently coboundaries). Let us assume the contrary, namely that the construction terminated, but some cell $a$ has element $b$ in its boundary. But then the Morse pairing between $a$ and $b$ could have been made, which gives a contradiction.

Let us now assume that we obtained a Morse complex with each cell having empty boundary and coboundary. From algebraic Morse theory we know that its homology is isomorphic to the homology of the initial complex. Homology is defined as the quotient $H_{n}:=\operatorname{ker} \partial_{n} / \mathrm{im} \partial_{n+1}$. Since all the boundary maps $\partial_{n}$ are zero, the image is empty and the kernel group is generated by all the cells. As a result, every cell generates a homology class and consequently $\beta_{n}(S)=\left|S_{n}\right|$.

It might seem that this algorithm contradicts some known hardness results related to constructing optimal gradient vector fields (Morse matchings) [10]. Indeed constructing such a matching on the input complex would imply that homology can be read from the related Morse complex. But we only define the optimal matching on the penultimate complex, and this cannot be transferred back to the original complex.

We will not discuss the complexity of this algorithm. Let us just state that using efficient algorithms to compute Morse complexes from [9]6] we get a bound of $O\left(n^{3}\right)$, where $\mathrm{n}$ is the size of the input complex. Experiments show that in practice the runtime is better.

In Algorithm 3 we describe the compression of the initial complex to a Morse complex. The procedure doMorsePairings $(C, d)$ performs Morse pairings in a 
complex $C$ under a constraint that the dimension of every element in a pairing is $\leq d$. The procedure computeMorseComplex $(C, V)$ computes the boundary coefficients between critical cells (see 78, for the theory and also 9] for algorithmic details) and removes from $C$ all the non-critical elements (i.e. elements from $V$ ). The procedure stops execution when there are no more pairings to be done in the complex. We want to point out that only the $d-2$ dimensional skeleton of the constructed complex is modified, because we need the simplices in dimension $d-1$ and $d$ in order to build the higher dimensional skeleton. This algorithm should be called for each step of construction in Algorithm 2,

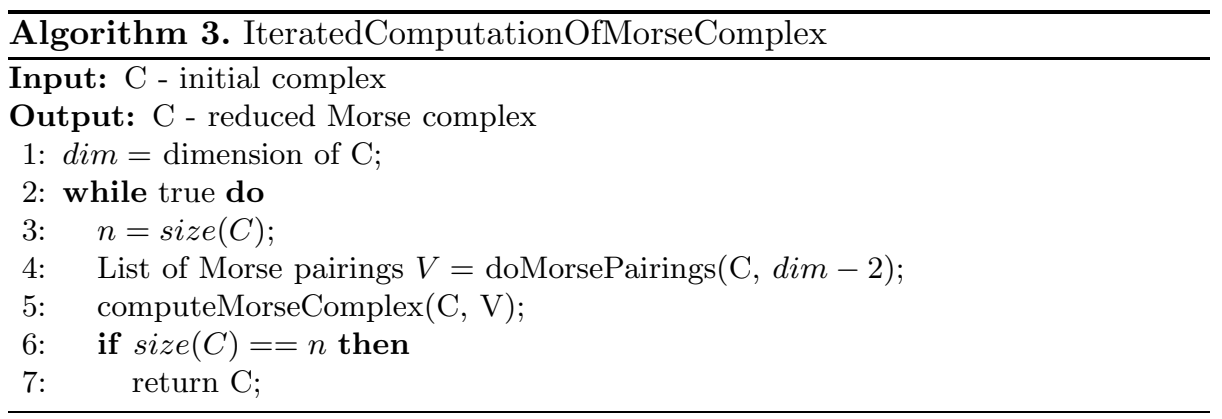

\section{Experiments}

We have developed and tested a $\mathrm{C}++$ implementation which includes the algorithms outlined above. To compute homology and persistent homology, we use the standard matrix reduction method [5], with the twist by Chen and Kerber [4]. For experiments we sample the corpus of the English Wikipedia [17], processed using Python library gensim [18].

There are two main parameters of our software. Parameter dim controls the maximum dimension of the constructed complex. As a result, homology is computed up to dimension dim -1 . The second parameter is $\epsilon \in[0,1]$, which means that only edges $(a, b)$ with $\operatorname{sim}(a, b) \geq 1-\epsilon$ are included in the skeleton. These parameters reduce the amounts of computations.

While in the worst case computing persistent homology takes cubic time, the reduction algorithm is typically assumed to take linear time in practical situations [4. Judging from our experiments, the behavior is definitely superlinear, probably roughly quadratic (in the size of the complex) for dimensions $\geq 3$ (see Figure 2left). For dimension $<2$ the time required to build the complex dominated over the persistence computations. Therefore, for higher dimensions the reduction algorithm is clearly the computational bottleneck. Additionally the number of cells grows super-linearly in the input size, but it is strongly dependent on the chosen $\epsilon$.

The observed quadratic behavior is important in the context of finding efficient methods of computing persistent homology. Recent research suggests that 

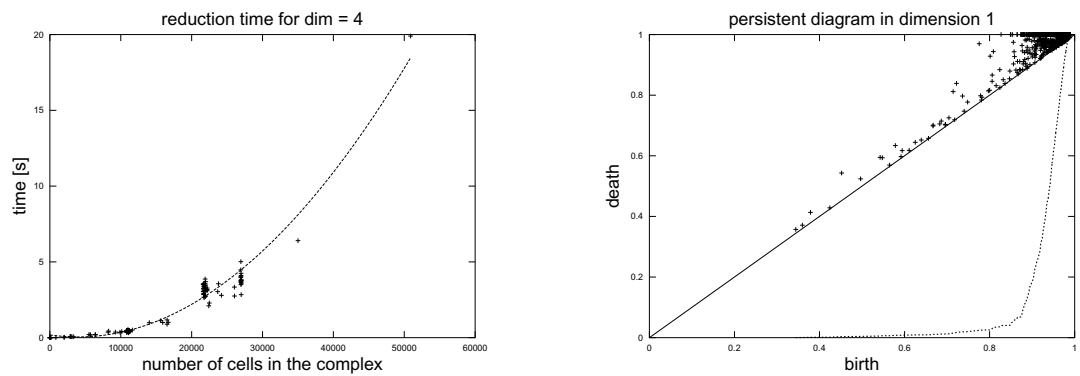

Fig. 2. Left: Runtime (in seconds) of the reduction algorithm for dimension 4. The behaviour appears to be quadratic, which is emphasised by fitting a degree two polynomial. Right: Persistence diagram for a complex containing $0.8 \mathrm{M}$ cells. We can assume 1 for infinity, since we know that at this point the skeleton would become a complete graph with all the cliques present. The curve in the lower part of the diagram represents a normalized cumulative sum of persistence values. It helps visualize the region of values for which features of relatively large persistence appear.

simplifying the input complex using discrete Morse theory (in a way which retains persistence) can increase efficiency. While in practice the significant advantage is in terms of memory usage [16, recent experiments show that such a simplification can minimize the problem related to the slow matrix reduction computation.

The observed quadratic behaviour is a good motivation for further development of algorithms based on discrete Morse theory. In case of textual data, such methods could help increase both the memory and time efficiency of persistence computations. Unfortunately, currently published methods, giving optimality in terms of the number of critical cells extracted, are limited to dimension $\leq 3$.

Our attempt to compute standard homology using discrete Morse theory was not very effective as the reduction factor was only about $10-20 \%$. This is probably related to the fact that we have many cycles in the highest dimension. We plan to investigate this issue further.

As shown in Figure 2 right, the 1-dimensional topology is quite uninteresting until the filtration value around 0.8 . It means that only after introducing edges with similarity $\leq 0.2$, do many features of larger persistence start to appear. On the other hand, we measured that the highest-dimensional cells are the most abundant in the complex. It appears that cells rarely cluster to create homological features of non-zero persistence. Experiments in higher dimensions (but for much smaller datasets) back up this statement. Note that in our setting a boundary of a single simplex either remains a cycle 'forever' (which actually means it is killed at value 1 , so persistence equals 1 -birth) or is filled instantly (zero persistence).

These observations suggest that features of non-zero persistence capture, let us call it, semi-similar sets of documents. By that we mean sets of documents 
which are similar enough to create a, say, $p$-dimensional cycle, but for a given threshold, they cannot fill this cycle. But, for a higher threshold, there can exist additional cells (related to additional documents) which do fill the cycle. Therefore, persistence can be viewed as the measure of discrepancy between the inter-similarity of a set of documents, and its certain superset.

Analysis of the 1-dimensional persistence suggests two explanations of the low number of features of non-zero persistence. 1) The similarities are very strong, many large cliques appear and most lower-dimensional features have zero persistence. 2) The similarities are strong only locally - almost all appearing cycles are boundaries of a single simplex, so their persistence is 0 (if they are killed). To verify this hypothesis experiments in higher $(>4)$ dimensions should be run.

\section{Summary}

The main purpose of this paper is to challenge the current computational topology tools with large text datasets represented within the vector-space model. The experimental results show that these methods lack efficiency. Specifically, the algorithm for persistent homology exhibits quadratic behaviour in the size of the constructed complex, which prevents our approach from scaling for realistic amounts of data. Interestingly, to the best of our knowledge, this is the first dataset exhibiting quadratic scaling, which comes from an application.

The experiments we were able to conduct did shed some light on the lowerdimensional topological structure of the dataset. In our future research we would like to answer some of the questions posed in the previous section as well as try to verify the efficiency of different computational methods.

Acknowledgments. All authors are supported by Google Research Awards programme. H.W. is also supported by a Foundation for Polish Science IPP Programme "Geometry and Topology in Physical Models". M.M. is partially supported by Polish MNSzW Grant N N201 419639, P.D. also by Grant Nr IP 2010046370.

\section{References}

1. Baeza-Yates, R., Ribeiro-Neto, B.: Modern information retrieval, p. 192. AddisonWesley Longman, Reading (1999)

2. Beygelzimer, A., Kakade, S., Langford, J.: Cover trees for nearest neighbor. In: Proc. of ICML 2006 (2006)

3. Carlson, G.: Topology and Data. Bulletin of the AMS 46(2), 255-308 (2009)

4. Chen, C., Kerber, M.: Persistent homology computation with a twist. In: 27th European Workshop on Computational Geometry, EuroCG 2011 (2011)

5. Edelsbrunner, H., Harer, J.L.: Computational Topology. An Introduction. Amer. Math. Soc., Providence (2010)

6. Feng, A.-X., Fu, C.-H., Xu, X.-L., Liu, A.-F., Chang, H., He, D.-R., Feng, G.-L.: An Empirical Investigation on Important Subgraphs in Cooperation-Competition networks. Science (2011) 
7. Forman, R.: A User's Guide To Discrete Morse Theory. Séminaire Lotharingien de Combinatoire B48c, 1-35 (2002)

8. Kozlov, D.: Combinatorial Algebraic Topology. Springer (2007)

9. Lewiner, T.: Geometric discrete Morse complexes, PhD Thesis (2005)

10. Lewiner, T., Lopes, H., Tavares, G.: Toward Optimality in Discrete Morse Theory. Experiment. Math. 12(3), 271-286 (2003)

11. Polanco, X., Juan, E.S.: Text Data Network Analysis Using Graph Approach. In: Proc. of InSciT, pp. 586-592 (2006)

12. Robins, V., Wood, P.J., Sheppard, A.P.: Theory and Algorithms for Constructing Discrete Morse Complexes from Grayscale Digital Images. IEEE Trans. Pattern Anal. Mach. Intell. 33, 1646-1658 (2011)

13. Salton, G., Wong, A., Yang, C.S.: A vector space model for automatic indexing. Commun. ACM 18(11), 613-620 (1975)

14. Zipf, G.K.: Human Behavior and the Principle of Least Effort. Addison-Wesley, Cambridge (1949)

15. Zomorodian, A.: Fast construction of the Vietoris-Rips complex. Computers \& Graphics 34(3), 263-271 (2010)

16. Günther, D., Reininghaus, J., Wagner, H., Hotz, I.: Memory Efficient Computation of Persistent Homology for 3D Image Data using Discrete Morse Theory. In: Sibgrapi 2011, Maceio, Brazil (2011)

17. English Wikipedia corpus, http://dumps.wikimedia.org/enwiki/

18. Gensim Library, http://radimrehurek.com/gensim/ 